\title{
Treating transgenic Alzheimer mice with a $\beta$-secretase inhibitor, what have we learned?
}

\author{
Jordan Tang ${ }^{1}$ and Arun Ghosh ${ }^{2}$ \\ ${ }^{1}$ Protein Studies Program, Oklahoma Medical Research Foundation, University of Oklahoma Health Science \\ Center, Oklahoma City, Oklahoma, USA \\ ${ }^{2}$ Departments of Chemistry and Medicinal Chemistry, Purdue University, West Lafayette, Indiana, USA
}

Commentary on: Chang et al. B-Secretase inhibitor GRL-8234 rescues age-related cognitive decline in APP transgenic mice. FASEB J. 2010; article fj.10-167213.

Received: 1/12/11; Accepted 1/17/11; Published: 1/17/11

Correspondence with Jordan Tang, PhD to Jordan-Tang@omrf.org.

(C) Tang and Ghosh. This is an open-access article distributed under the terms of the Creative Commons Attribution License, which permits unrestricted use, distribution, and reproduction in any medium, provided the original author and source are credited.

$\beta$-Secretase is an attractive target of amyloid-reduction therapy for Alzheimer's disease. Currently, no efficacy data is available from clinical trials of $\beta$-secretase inhibitors. Treating young transgenic Tg2576 mice with a brain-penetrating $\beta$-secretase inhibitor reduced brain amyloid- $\beta$ by about $50 \%$ and rescued the age-related cognitive decline. Implications from these model studies on the design of clinical trials are discussed herein.

Since the identification of $\beta$-secretase (memapsin 2, BACE1) more than a decade ago, significant research effort from many laboratories around the world has been directed towards the development of inhibitor drugs against this $\beta$-secretase as a potential treatment of Alzheimer's disease (AD) [1]. The initial excitement and optimism were understandable. Since amyloid- $\beta$ $(\mathrm{A} \beta)$ is an important driving force in $\mathrm{AD}$ pathogenesis, clinical intervention to reduce its production would be a logical therapeutic approach. Toward this end, the inhibition of $\beta$ - or $\gamma$-secretase are the best choices for drug development, as these two proteases represent the first and second steps in the processing of amyloid precursor protein leading to $A \beta$ production.

Inhibitor drugs for $\gamma$-secretase have exhibited toxicity problems in several clinical trials which appear to be rooted in the many important physiological functions of this protease, and possibly in the lack of alternative pathways to process its natural substrates when $\gamma$ secretase is inhibited. These are fundamental problems inherent to this target and have evaded practical solutions thus far. $\beta$-Secretase, on the other hand, is devoid of many of these problems. The inhibition of this first step in $A \beta$ production may avoid other harmful late events in AD pathogenesis. Deletion of the $\beta$-secretase gene to eliminate $A \beta$ production resulted in only minor phenotype changes in mice, suggesting that a partial reduction of $A \beta$ generation by $\beta$-secretase inhibitors could be safe in clinical treatments. In addition, as an aspartic protease, $\beta$-secretase enjoys a successful precedence in the development of HIV protease inhibitor drugs used today.

The attractiveness of $\beta$-secretase as a drug target, however, has not translated into rapid pharmaceutical development. A decade after the target discovery, only a few drug candidates have entered the early phases of clinical trials and no successful demonstration of efficacy has been reported in human trials. The slow pace of pharmaceutical development coupled with the suspension of a number of $\beta$-secretase inhibitor projects in major companies, have earned $\beta$-secretase a perhaps deserving reputation of being a difficult target. In other words, it has been difficult to develop $\beta$-secretase inhibitors that possess all required properties such as blood-brain barrier penetration, selectivity, pharmacokinetic characteristics and toxicity. To a large extent, these difficulties are unique to this target. $\beta$-Secretase has a large active-site area for inhibitor binding. Yet it permits relatively limited options for designing small, potent and selective inhibitors and thus renders 
ineffective some of the traditional drug discovery tools, such as automated screening of chemical libraries or computer assisted inhibitor design.

However, by using crystal structure based inhibitor design, more drug-like inhibitors have emerged. At least three pharmaceutical companies have conducted Phase I trials for their inhibitor candidates; but convincing efficacy results from clinical trials will take years of future efforts. It is therefore appropriate to ask, what we have learned from therapeutic model, such as a transgenic $\mathrm{AD}$ mouse with $\beta$-secretase inhibitors? Although AD mice do not accurately duplicate human $\mathrm{AD}$, they do share some early human $\mathrm{AD}$ features, which include excess production of brain $A \beta$, a decline of cognitive performance with age, and the development of amyloid plaques in the brain at middle-late age. $\mathrm{AD}$ mice do not progress to dementia as in human $\mathrm{AD}$. However, for testing an amyloid reduction therapy using $\beta$-secretase inhibitors, the $\mathrm{AD}$ syndromes in these mice can produce useful insights to human treatments. At this stage in $\beta$-secretase inhibitor development, it would be informative to learn if these AD-like syndromes can be rescued in $\mathrm{AD}$ mice by partial amyloid reduction.

Chang et al. reported recently [2] that the administration of $\beta$-secretase inhibitor GRL-8234 rescued age-related cognitive decline and reduced brain amyloid burden in transgenic $\mathrm{AD}$ mice $\mathrm{Tg} 2576$. In this mouse strain, excess $A \beta$ produced in the brain leads to cognitive decline starting at about 6 months of age and continues through life. At about 10 months of age, amyloid plaques start to appear in the brain. The age-related cognitive decline in this strain of AD mice [3], which is dependent on the overproduction of $A \beta$ in the brain, is the most stringent efficacy criteria available in $\mathrm{AD}$ mouse model. In the studies of Chang et al., AD mice were continuously infused with GRL-8234 which reduced brain and plasma $A \beta$ by about $50 \%$ of the controls. In three separate experiments in which the starting age of the mice ranged from 5.5 to 9 months, differences in the cognitive performance between the treated and the control mice were seen only after a period ranging from about 4 to 7 months. Treatments of shorter periods or starting treatment at an older age (18 months) failed to show the benefit of cognitive rescue. In all the experiments which resulted in cognitive rescue, the reduction of brain amyloid was about $50 \%$.

These results are encouraging and several interesting points have emerged from this study. First, a partial reduction of $\mathrm{A} \beta$ production and brain plaque load of about $50 \%$ was sufficient to rescue the cognitive decline in this model. The current results appear to suggest that the cognitive decline can be significantly improved in spite of some excess of brain $A \beta$. Conceivable mechanisms to accommodate these findings may include the presence of an $A \beta$ threshold for cognitive decline or partial $A \beta$ reduction may delay the onset of cognitive decline. Regardless of the mechanism, efficacy of cognitive rescue by partial $A \beta$ reduction would be good news if it is confirmed by clinical studies. Second, there was no overt toxicity associated with treatment over a 7 month time period and no significant accumulation of APP in the brain of treated mice, a potential mechanism based side effect. The latter observation suggests that the known alternative APP processing pathway by $\alpha$-secretase was sufficiently active to dispose an overproduced $\beta$ secretase substrate. Third, it took several months, which was somewhat surprising to manifest cognitive rescue in inhibitor treated mice. One explanation is that the cognitive decline in $\mathrm{Tg} 2576$ is known to be a slow process. Thus, the differential of cognitive performance between the treated and control groups would require a long time. Fourth, inhibitor treatment of old mice about 6 months after the appearance of brain plaque failed to show the benefit. This failure may stem from the fact that the old mice became incompetent for cognitive tests before the rescue could be demonstrated. Another possibility is that some of the amyloid existed in the brain of old mice prior to the beginning of the treatment may serve as a source of $A \beta$ in a partial resolubilization process. This may have kept $A \beta$ levels higher than expected and thus would require an even longer time to show cognitive rescue. It is perhaps worthwhile to consider the last two points in the setting of human clinical trials. In AD patients, cognitive performance declines slowly in general and amyloid plaques are present in the later stages of the disease. For clinical trials of $\beta$-secretase inhibitor drugs, cognitive tests are the only efficacy criteria acceptable for the FDA. Therefore, a Phase II/III trial with early stage AD patients over a long trial period will have the best chance to avoid the potential problems discussed above. In addition, testing $\beta$-secretase inhibitor drugs in an amyloid reduction concept, early stage $\mathrm{AD}$ patients would have less brain destruction and inflammation, which is not expected to be reversed quickly by $\beta$ secretase inhibitors and may obscure the benefit of the drugs. It will be of interest to study the same $\beta$-secretase inhibitor in both $\mathrm{AD}$ mice and human efficacy trials. A predictive model may contribute to the planning and decisions on long, costly human trials.

\section{REFERENCES}

1. Ghosh AK, Gemma S, Tang J. beta-Secretase as a Therapeutic Target for Alzheimer's Disease. Neurotherapeu- 
tics 2008; 5: 399-408.

2. Chang $W$, Huang $X$, Downs $D$, Cirrito J, Koelsch $G$, Holzman DM, Ghosh AK, Tang J. $\beta$-Secretase inhibitor GRL-8234 rescues age-related cognitive decline in APP transgenic mice. FASEB J. 2010; article fj.10-167213. Epub ahead of print: November 8, 2010.

3. Lesne $S$, Koh MT, Kotilinek L, Kayed R, Glabe CG,Yang A, Gallagher $\mathrm{M}$, Ashe K H A specific amyloid-beta protein assembly in the brain impairs memory. Nature 2006; 440: 352-357. 